\title{
COSTA RICA: RECONFIGURACIÓN POLÍTICA EN UN CONTEXTO DE GOBIERNO DIVIDIDO*
}

\author{
Costa Rica: Political reconfiguration under divided government
}

\section{RONALD ALFARO-REDONDO}

Programa Estado de la Nación

\section{STEFFAN GÓMEZ-CAMPOS}

Programa Estado de la Nación

\begin{abstract}
RESUMEN
La dinámica sociopolítica de Costa Rica en 2010-2011 se puede resumir en tres grandes áreas. En lo político, la gestión del gobierno ha estado marcada por divisiones internas, una alta rotación de ministros, y un fuerte desgaste en sus relaciones con el Legislativo. En materia económica hay una recuperación moderada y frágil, que se da en medio de la discusión sobre la difícil situación fiscal del país. Y en términos sociales, el panorama general es paradójico: por un lado se consolidan fortalezas en indicadores como esperanza de vida; y altas coberturas educativas y de la seguridad social, pero por el otro, se deterioran aspectos como el aumento en la desigualdad; la criminalidad y las dificultades para ejecutar obra pública.
\end{abstract}

Palabras clave: Costa Rica, elecciones, gestión política, crisis económica, relaciones Ejecutivo y Legislativo.

\begin{abstract}
Three main features characterize socio-political events in Costa Rica during 2010 and 2011. First, in the political system, internal divisions within the incumbent party and across other parties in Congress, the constant reshuffles in the cabinet under divided government have challenged the new government. S 1'SXSSSSSecond, the economic situation is improving after the most recent crisis, despite weaknesses remains. Finally, in the social realm there is a paradox, on one hand, the traditional human development indicators (life expectancy, education and social security) have improved over time whereas on the other, there have been an increasing trend in social inequality, and crime as well as a worsening in the public capacities to build required infrastructure.
\end{abstract}

Key words: Costa Rica, elections, political management, economic crisis, Legislative and Executive.

* Los autores agradecen a Juan Guillermo Murillo del Programa Estado de la Nación por su colaboración en la preparación de insumos para este artículo. 


\section{TEMAS RELEVANTES DE LA REALIDAD NACIONAL Y SU IMPACTO POLÍTICO}

El presente artículo analiza la situación política en Costa Rica durante el año 2010 y el 2011. El período bajo estudio comprende de enero a diciembre en ambos años. El documento analiza las principales repercusiones de los cambios socio-políticos más recientes y examine en detalle las complejas situaciones a las que hace frente el actual gobierno. Se profundiza en aspectos políticos, económicos y sociales.

Las principales conclusiones son las siguientes: a pesar de haber obtenido una holgada victoria electoral en febrero del 2010, la administración Chinchilla Miranda (20102014) ha enfrentado dificultades externas e internas que han obstaculizado su agenda de gobierno. En el plano interno sobresalen las divisiones en su fracción legislativa, los constantes movimientos en su gabinete y la ausencia de mayoría en el Congreso. Además el conflicto con Nicaragua ha concentrado los esfuerzos de la administración en política exterior. En el ámbito político electoral, destacan el aumento en la participación política, el incremento de la fragmentación del sistema de partidos y la disputa por el control de los municipios. En otra materia, el desempeño de la economía durante los últimos dos años volvió a ser positivo, sin embargo se incrementó la desigualdad y aumentó la criminalidad.

\subsection{Aspectos políticos}

El 2010 y 2011 estuvieron marcados por eventos que reflejan las dificultades en torno a la gestión política de asuntos prioritarios para el país, e importantes divisiones entre los principales partidos políticos representados en el Legislativo, que entorpecen el diálogo y la construcción de acuerdos. En el Congreso, una coalición de partidos de oposición tomó el control del Directorio Legislativo para el periodo 2011-2012 y dio paso a la configuración de un gobierno dividido, un hecho inédito desde la década de los años 1970. Las pugnas por el control del Directorio llevaron a otra situación poco común en la historia reciente del país: por primera vez en 75 años un Jefe de Estado no logra dar su informe anual de labores al Congreso el 01 de mayo.

Con el Directorio Legislativo en manos de la oposición, al Ejecutivo se le ha dificultado avanzar en la aprobación de proyectos de ley prioritarios, entre los que destaca el Plan Fiscal, que constituye la iniciativa más importante para el oficialismo desde el inicio de la administración Chinchilla.

En el plano municipal, durante el primer año de gestión de las nuevas autoridades locales que se renovaron en el 2010, se celebró por primera vez un plebiscito revocatorio cuyo resultado fue la destitución del alcalde del cantón de Pérez Zeledón. Además, las diferencias entre alcaldes y regidores, junto con la falta de planificación generaron el rechazo del presupuesto ordinario en nueve municipalidades, por parte de la Contraloría General de la República. Y en materia de control de la corrupción, destaca la apertura de al menos cuatro denuncias penales, tres procesos en estrados judiciales y 21 trámites de sanción administrativa contra diferentes alcaldes. Las investigaciones atienden presuntos actos de corrupción, uso indebido de los recursos 
públicos, incumplimiento de trámites legales y falta de fiscalización de los fondos municipales, entre otros aspectos.

La situación subregional por su parte, estuvo dominada por el conflicto de Costa Rica con Nicaragua cuando este último país inició, en octubre de 2010, obras de dragado en el río San Juan. Nicaragua procedió con los trabajos sin informar a Costa Rica sobre los detalles técnicos del proyecto, ni sobre eventuales impactos negativos en los humedales que conforman la parte norte fronteriza del territorio costarricense denominado Isla Portillos-Isla Calero y el delta Colorado-San Juan. Posteriormente se corroboró que las personas encargadas del dragado estaban construyendo un caño y depositando los sedimentos en territorio nacional, en una zona que había sido inscrita por Costa Rica en la lista de humedales de importancia internacional bajo la Convención Ramsar. Además se evidenció que las fuerzas armadas nicaragüenses cruzaron la frontera y ocuparon territorio costarricense, atentando así contra la soberanía nacional.

Motivado por tales hechos, el gobierno acudió primero a la Organización de Estados Americanos (OEA) donde denunció la ocupación de territorio nacional por fuerzas armadas nicaragüenses. En ese Foro veintiún países miembros solicitaron a Nicaragua, entre otras cosas, el retiro de sus tropas. Sin embargo, el gobierno nicaragüense hizo caso omiso y continuó con el dragado del río San Juan. Como segunda medida, en noviembre de Costa Rica demandó a Nicaragua ante la Corte Interamericana de Justicia (CIJ), por invadir su territorio y por la destrucción de los humedales de la zona. A inicios del 2011 la CIJ estableció medidas provisionales y ordenó a ambas partes abstenerse de "enviar a, o mantener en el territorio en disputa, incluyendo el 'caño', cualquier personal, sea civil, policía o de seguridad", y resolvió que solo Costa Rica puede enviar a la zona personal civil encargado de la protección ambiental en consulta con la Secretaría de la Convención Ramsar.

\subsection{Aspectos económicos}

El desempeño económico durante del país en los últimos dos años volvió a ser positivo, luego de una tasa de crecimiento de -1\% del PIB durante el 2009. En 2010 el Producto Interno Bruto (PIB) del país creció al 4,7\% y durante el 2011 al 4,2\%. El motor de este crecimiento fue, por tercer año consecutivo, la demanda interna, y aunque la demanda externa se ha recuperado, no ha logrado establecerse en los niveles que tenía previo al periodo de crisis que inició en el 2008.

No obstante, este crecimiento es moderado y frágil. Ello se refleja en un modesto comportamiento de la producción y en el estancamiento de la generación de oportunidades durante los últimos dos años. El desempleo se mantuvo elevado (7,3\% en el 2010 y 7,7\% en 2011), con mayor incremento en la zona urbana. Sectores como el agro, enseñanza y turismo, que en el 2010 generaron nuevos puestos de trabajo, en el 2011 mostraron reducciones en el número de ocupados. Por su parte, las actividades de comercio, construcción y comunicaciones que venían con resultados negativos en el 2010, fueron una importante fuente de empleo para el 2011 (Programa Estado de la Nación, 2011).

Otros resultados fueron más bien positivos. La inflación se mantuvo baja por tercer año consecutivo (por debajo del 6\%), y las exportaciones volvieron a crecer durante el 
$2010(6,8 \%)$. Sin embargo surge el riesgo de un déficit fiscal cada vez mayor. El déficit promedio del gobierno central como proporción del PIB durante el periodo 1999-2009 fue de $-2,2 \%$, pero en el 2009 se observa un aumento sobre el cual, el gobierno ha llamado la atención (-3,4\% en 2009 y -5,3\% en 2010). Bajo esa coyuntura el Ejecutivo ha promovido en el Congreso, desde inicios de 2010, una reforma fiscal que permita identificar nuevas fuentes de recursos económicos, aunque sin éxito de aprobación hasta el momento. ${ }^{1}$

\subsection{Aspectos sociales}

Costa Rica goza de un conjunto de condiciones sociales que lo ubican dentro del grupo de países con alto Índice de Desarrollo Humano (IDH), ${ }^{2}$ de acuerdo con el Programa de Naciones Unidas para el Desarrollo (PNUD). En ese sentido, hay un grupo de indicadores que confirman la fortaleza del estado de bienestar costarricense. En particular una alta esperanza de vida (79 años), altas coberturas de la seguridad social (69,5\%), y las mejoras en las coberturas de educación en los últimos años (educación secundaria por encima del 70\%).

Sin embargo, hay un grupo de indicadores que emerge como serios riesgos para el desarrollo humano, entre los que destaca la pobreza, las crecientes brechas de desigualdad y la criminalidad entre otros. El país no ha logrado reducir de manera significativa la incidencia de la pobreza durante la última década. Actualmente se mantiene estancada en el $21 \%$, con diferencias según zona de residencia. Las áreas urbanas tienen menor incidencia de pobreza respecto a las rurales: $19,1 \%$ y $26,0 \%$ respectivamente.

La desigualdad es otro de los elementos con cambios significativos en los últimos años. La evidencia muestra que el país tiende a un incremento en la desigualdad, medida por el coeficiente de Gini y por la diferencia de ingresos de los hogares. Por ejemplo, el Coeficiente de Gini que mide la distribución del ingreso per cápita a nivel de hogares, era de 0,464 en 1990, y para el 2010 creció a 0,508. Por su parte la diferencia entre el ingreso del primer quintil de los hogares (el más pobre) y el quinto quintil (el más rico) fue de 11,6 veces en 2011, el más alto de la década (Cuadro 1).

\section{CAMBIOS INSTITUCIONALES Y EN POLÍTICAS PÚBLICAS}

Desde el 2010 se han dado pocos cambios sustanciales de tipo institucional o en políticas públicas. Debe considerarse que la aprobación de la legislación sustantiva toma en promedio más de dos años, como se explica más adelante, con lo cual el gobierno entrante podría ver materializadas algunas de sus principales iniciativas sólo a partir del 2012. A pesar de ello, entre 2010 y 2011 se pueden citar al menos tres cambios de importancia.

1 Al momento de redacción de este documento el Plan Fiscal, aprobado en primer debate por el Congreso, fue declarado inconstitucional por la Sala Constitucional, por tener vicios de procedimiento en el proceso de aprobación legislativa.

2 El país ocupa el lugar 7 en América Latina, por debajo de Chile, Argentina, Uruguay, Cuba, México y Panamá; y el 69 del mundo según la última medición del PNUD de 2011. 
Cuadro 1. Costa Rica: Principales indicadores económicos y sociales 2000-2011

\begin{tabular}{|c|c|c|c|c|c|c|c|c|}
\hline Indicador & 2000 & 2005 & 2006 & 2007 & 2008 & 2009 & 2010 & 2011 \\
\hline $\begin{array}{l}\text { Ingreso nacional bruto (INB) per cápita } \\
\text { (Constante } 2005, \$ \text { internacionales) }^{\mathrm{a}}\end{array}$ & 7467 & 8650 & 9327 & 9933 & 10116 & 9917 & 10205 & 10497 \\
\hline Crecimiento del PIB (\%) & 1,8 & 5,9 & 8,8 & 7,9 & 2,7 & $-1,0$ & 4,7 & 4,2 \\
\hline $\begin{array}{l}\text { Crecimiento de las exportaciones } \\
\text { (\$ corrientes) }\end{array}$ & $-12,2$ & 11,5 & 16,7 & 13,9 & 1,8 & $-7,6$ & 6,8 & $\mathrm{~N} / \mathrm{D}$ \\
\hline $\begin{array}{l}\text { Déficit/superávit Gobierno } \\
\text { Central/PIB (\%) }\end{array}$ & $-3,0$ & $-2,1$ & $-1,1$ & 0,6 & 0,2 & $-3,4$ & $-5,3$ & $\mathrm{~N} / \mathrm{D}$ \\
\hline Inflación (IPC) (\%) & 10,2 & 14,1 & 9,4 & 10,8 & 13,9 & 4,0 & 5,8 & $\mathrm{~N} / \mathrm{D}$ \\
\hline Tasa de desempleo abierto (\%) & 5,2 & 6,6 & 6,0 & 4,6 & 4,9 & 8,4 & 7,3 & 7,7 \\
\hline $\begin{array}{l}\text { Cobertura de la PEA por seguro } \\
\text { de salud }(\%)\end{array}$ & 64,63 & 55,0 & 57,8 & 61,9 & 66,5 & 64,8 & 70,0 & 69,5 \\
\hline $\begin{array}{l}\text { Cobertura promedio en educación } \\
\text { secundaria }(\%)\end{array}$ & $\mathrm{N} / \mathrm{D}$ & 67,2 & 69,3 & 69,5 & 69,5 & 71,9 & 72,9 & $\mathrm{~N} / \mathrm{D}$ \\
\hline Esperanza de vida al nacer (años) & 77,7 & 79,1 & 79,1 & 79,1 & 79,1 & 79,2 & 79,0 & $\mathrm{~N} / \mathrm{D}$ \\
\hline $\begin{array}{l}\text { Tasa de mortalidad infantil } \\
\text { (por } 1.000 \text { nacidos vivos) }\end{array}$ & 10,2 & 9,8 & 9,7 & 10,1 & 9,0 & 8,8 & 9,5 & $\mathrm{~N} / \mathrm{D}$ \\
\hline $\begin{array}{l}\text { Tasa de homicidios } \\
\text { (por cien mil habitantes) }\end{array}$ & 6,1 & 6,9 & 7,7 & 8,0 & 11,1 & 11,8 & 11,5 & $\mathrm{~N} / \mathrm{D}$ \\
\hline Hogares pobres $(\%)^{\mathrm{b}}$ & 20,6 & 21,2 & 20,2 & 16,7 & 17,7 & 18,5 & 21,3 & 21, \\
\hline Coeficiente de Ginib & 0,487 & 0,485 & 0,500 & 0,502 & 0,498 & 0,516 & 0,508 & $\mathrm{~N} / \mathrm{D}$ \\
\hline $\begin{array}{l}\text { Relación entre el ingreso promedio } \\
\text { del hogar del V quintil y del I quintil } \\
\left(\text { veces) }{ }^{c}\right.\end{array}$ & 9,7 & 9,4 & 10,0 & 9,6 & 9,0 & 10,1 & 9,9 & 11 \\
\hline
\end{tabular}

a Datos del Informe de Desarrollo Humano 2011, PNUD.

${ }^{\mathrm{b}}$ Estas variables tienen como fuente las Encuestas de Hogares (INEC). Las cifras del 2000 al 2009 corresponden a las Encuestas de Hogares de Propósitos Múltiples (EHPM) y son totalmente comparables entre ellas. Las cifras del 2010 proceden de la Encuesta Nacional de Hogares (ENAHO), por lo cual no son comparables con las de años anteriores debido a: cambios en la muestra y en el cuestionario; utilización de las nuevas proyecciones de población de septiembre del 2008 para la determinación de los factores de expansión; aumento en el límite de la edad de la fuerza de trabajo, que pasó de 12 a 15 años; y cambios en la medición del ingreso y de la pobreza.

${ }^{\mathrm{c}}$ Los quintiles son establecidos con base en el ingreso promedio per cápita del hogar. Para estimar los quintiles en el 2010, se utilizó el ingreso neto sin ajuste.

Fuente: Elaboración propia con datos del Programa Estado de la Nación, el Banco Central de Costa Rica, y el PNUD.

El primero de ellos tiene que ver con modificaciones para garantizar la participación política de las mujeres en diferentes puestos de la gestión pública. En este sentido, la Ley 8910 sobre el porcentaje mínimo de mujeres que deben integrar las directivas de asociaciones, sindicatos y asociaciones solidarias, aplica el principio de paridad de género en la conformación de tales juntas directivas. La ley indica que en los órganos o 
nóminas impares, la diferencia del total de hombres y mujeres no puede ser superior a uno. La aprobación se da un año después de que el Código Electoral de 2009 estableciera también el principio de paridad de género con el mecanismo de alternancia en las listas de elección popular y nóminas internas de los partidos políticos, para garantizar el $50 \%$ de representación femenina.

El otro aporte en materia de política pública fue la Política Integral y Sostenible de Seguridad Ciudadana y Promoción de la Paz Social (Polsepaz), que presentó el Ejecutivo a inicios del 2011. Esta es sin duda, una de las áreas que más requiere de la coordinación de esfuerzos institucionales y asignación de recursos para la ejecución de políticas concretas, pues el aumento de los indicadores de criminalidad han hecho de este un tema preocupante, a tal grado que en junio de 2011 un 46\% de la población identificó la inseguridad como el mayor problema del país (Villalobos, 2011). Sin embargo, Polsepaz constituye una iniciativa con poca o nula identificación programas y acciones específicas, sin definición de plazos ni asignaciones presupuestarias para su cumplimiento. Es más bien el producto de una amplia consulta nacional que describe la situación de delincuencia y marca áreas generales de acción.

El tercer cambio de importancia se aprobó en la Asamblea Legislativa en mayo de 2011 con la reforma del artículo 78 de la Constitución Política para el fortalecimiento del derecho a la educación. La normativa establece un incremento en la inversión estatal, incluida la superior, la cual no será inferior al 8\% anual del PIB. Esta disposición entraría a regir a partir del 2014. La Ley contempla que el gasto de cada año no podrá ser inferior al anterior, por lo que se espera un aumento gradual.

\section{EL 2010: UN AÑO ELECTORAL}

\subsection{El Partido Liberación Nacional y su dominio electoral}

El 7 de febrero del 2010, los costarricenses efectuaron el décimo quinto proceso electoral consecutivo desde 1953. En esta oportunidad se renovaron los cargos de Presidente y Vicepresidentes de la República, Diputados a la Asamblea Legislativa y concejales municipales en las 81 municipalidades del país. En total se designaron 1.050 cargos en todo el país. El Partido Liberación Nacional (PLN) -la agrupación más longeva- logró mantener bajo su control el Poder Ejecutivo por dos períodos consecutivos. Además cuenta con la fracción más numerosa en el Congreso -aunque sin alcanzar mayoría simpley la mayor cantidad de regidores en las municipalidades. Por otra parte, en diciembre del 2010 se celebraron los comicios de alcaldes municipales. Estas elecciones locales reafirmaron el amplio predominio del PLN en las alcaldías a pesar de la fragmentación partidaria en los Concejos Municipales.

De la contienda sobresalen cuatro aspectos importantes. Por primera vez en la historia del país una mujer ocupa el cargo de Presidente de la República. Asimismo, nuevas reglas electorales entraron a regir justo antes de la realización de los comicios. En términos generales las elecciones se desarrollaron en un ambiente de normalidad. Los comicios presidenciales se definieron por un amplio margen de diferencia, no así la elección 
parlamentaria cuyos resultados arrojaron un Congreso sin mayoría y con representación de ocho fuerzas políticas (Cuadro 2). Por último, el porcentaje de electores que se abstuvo de participar disminuyó por primera vez desde 1998.

\subsection{Se redujo el abstencionismo}

Un elemento novedoso de las elecciones generales 2010 fue el incremento de la participación electoral en $4 \%$ con respecto al 2006. Con ello se interrumpe la tendencia creciente del abstencionismo observada en las tres elecciones del periodo 1998-2006. A pesar de ello, un patrón que permanece invariable desde 1953 es la alta ausencia de electores en las tres provincias periféricas. Guanacaste, Puntarenas y Limón han sido históricamente y continúan siéndolo, las provincias en donde menos se ejerce el sufragio. Estas provincias son a su vez las regiones de menores niveles de desarrollo y menores oportunidades para sus pobladores, lo cual reafirma la existencia de desigualdades y brechas políticas que se manifiestan regionalmente.

El Partido Liberación Nacional (PLN) fundado en 1951, de tradición socialdemócrata y hoy día catalogado como centrista, ganó la elección con el 47\% de los votos. En segundo lugar se ubicó el Partido Acción Ciudadana (PAC, creado en el 2001) de inclinación centroizquierda con un distante 25\%. En tercera posición se situó el Movimiento Libertario (ML) con el 21\% de los votos, una agrupación de derecha que participa por cuarta ocasión. El Partido Unidad Social Cristiana (PUSC) de centro-derecha y segunda fuerza política desde 1986 alcanzó únicamente el 4\%.

En términos generales, las elecciones del 2010 no cambiaron radicalmente el panorama incierto en cuanto a la recomposición del sistema de partidos en nuestro país. Por el contrario, el impasse abierto en 2006 luego del desplome en el respaldo electoral del

Cuadro 2: Resumen de resultados de la elección general 2010

\begin{tabular}{lccccccc}
\hline \multirow{2}{*}{\multicolumn{1}{c}{ Partido Político }} & \multicolumn{2}{c}{ Elección Presidencial } & & \multicolumn{2}{c}{ Elección Legislativa } & \multirow{2}{*}{ Escaños } \\
\cline { 2 - 3 } & Absolutos & $\%$ & & Absolutos & $\%$ & \\
\hline Liberación Nacional & 896,516 & 46.9 & & 708,043 & 37.3 & 24 \\
Acción Ciudadana & 478,877 & 25.1 & & 334,636 & 17.6 & 11 \\
Movimiento Libertario & 399,778 & 20.9 & & 275,518 & 14.5 & 9 \\
Unidad Social Cristiana & 74,114 & 3.9 & & 155,047 & 8.2 & 6 \\
Otros partidos & 62,038 & 3.2 & & 426,581 & 22.5 & 7 \\
\hline Total votos válidos & $1,911,333$ & 100,0 & & $1,899,825$ & 100,0 & 57 \\
\hline
\end{tabular}

a La categoría "Otros partidos" incluye en la elección presidencial a los restantes cinco partidos políticos, cuatro de los cuales no alcanzaron siquiera el 1\% de los votos válidos emitidos. En el caso de la elección legislativa, dicha categoría agrupa a los restantes 14 partidos políticos, uno de los cuales obtiene cuatro diputados y tres de los cuales obtienen un diputado.

Fuente: Elaboración propia con información del TSE. 
Partido Unidad Social Cristiana y el posicionamiento del PAC como segunda fuerza política se prolonga en el tiempo y por lo tanto, se corrobora con ello la etapa de transición del sistema de partidos hacia un nuevo e indefinido estadio.

\subsection{PLN sin claro predominio en el Congreso}

En comparación con la composición parlamentaria del 2006, la fracción de Liberación Nacional disminuyó de 25 a 24 diputados, el PAC de 17 a 11, el Movimiento Libertario obtuvo 9 legisladores, el PUSC incrementó su fracción de 5 a 6 parlamentarios, el Partido Accesibilidad sin Exclusión (PASE) pasó de 1 a 4 , y los restantes 3 partidos que obtuvieron representación legislativa son unipersonales ${ }^{3}$. Por quinta elección consecutiva desde 1994, ninguna agrupación política cuenta con mayoría parlamentaria (29 diputados), lo cual obliga a todas las fuerzas a formas coaliciones para la aprobación de leyes.

La Asamblea Legislativa 2010-2014 se caracteriza por ser, junto con las del período 19741978 y 2006-2010, las de mayor fragmentación dada la representación de 8 partidos políticos. El antecedente más cercano en esta materia se dio en el período 1998-2002 en el que 7 partidos se distribuyeron los 57 escaños.

La composición histórica del Congreso (1953-2010) por familias de partidos políticos ${ }^{4}$ muestra la concentración de los escaños legislativos en los partidos de centro-centro/ derecha. A pesar del repunte de las agrupaciones de izquierda y centro/izquierda en 2006, en 2010 las fuerzas del polo ideológico predominante en la política costarricense recuperaron una proporción importante de sus curules (Cuadro 3).

\subsection{Predomina la fragmentación política a nivel municipal}

En lo que respecta a las elecciones de regidores municipales, el 2010 es la última elección local concurrente con la elección presidencial y legislativa, pues en 2016 se unificarán todas las elecciones municipales en una misma fecha, justo dos años después de la elección nacional.

En 2010 el Partido Liberación Nacional obtuvo 196 concejales municipales (el 40\%) de un total de 495 en todo el país. Le sigue el Partido Acción Ciudadana con 99, equivalente al 20\%. Por debajo del PLN y el PAC se ubican el Movimiento Libertario y el Partido Unidad Social Cristiana con $75(15 \%)$ y $53(11 \%)$ plazas respectivamente. El Partido Accesibilidad sin Exclusión (PASE) obtuvo 23 regidores (5\%). Luego aparecen otras 40 agrupaciones que obtienen cuatro plazas o menos en las municipalidades.

\footnotetext{
Se trata de los casos del Frente Amplio (FA), Restauración Costarricense (PRC) y Restauración Nacional (PRN). El concepto de familias de partidos políticos (Sánchez, 2004) agrupa a la amplia gama de agrupaciones políticas que compiten en los diferentes procesos electorales en tres categorías: 1) el Partido Liberación Nacional (que agrupa a un solo partido, denominada Partido de centro), 2) los partidos de la Alianza Conservadora (llamada Partidos de derecha y centro/derecha) y 3) los partidos de izquierda (Partidos de izquierda y centro/izquierda). En este documento se agrega una categoría más a las formuladas por Sánchez dada la especificidad de las agrupaciones que la componen, denominada 4) Partidos regionales e intereses específicos.
} 
Cuadro 3: Composición de la Asamblea Legislativa según familia de partidos políticos 1953-2010

\begin{tabular}{|c|c|c|c|c|c|c|c|c|c|c|c|c|c|c|c|}
\hline $\begin{array}{l}\text { Familia de } \\
\text { partidos políticos }^{\mathrm{a}}\end{array}$ & $1953^{\prime}$ & $58^{\prime}$ & $62^{\prime}$ & $66^{\prime}$ & $70^{\prime}$ & $74^{\prime}$ & $78^{\prime}$ & $82^{\prime}$ & $86^{\prime}$ & $90^{\prime}$ & $94^{\prime}$ & $98^{\prime}$ & $02^{\prime}$ & $06^{\prime}$ & $10^{\prime}$ \\
\hline Partido de centro & 30 & 20 & 29 & 29 & 32 & 27 & 25 & 33 & 29 & 25 & 28 & 23 & 17 & 25 & 24 \\
\hline $\begin{array}{l}\text { Partidos de derecha y } \\
\text { centro/derecha }\end{array}$ & 15 & 25 & 27 & 28 & 23 & 26 & 27 & 19 & 25 & 29 & 25 & 28 & 25 & 12 & 15 \\
\hline $\begin{array}{l}\text { Partidos de izquierda y } \\
\text { centro/izquierda }\end{array}$ & 0 & 0 & 1 & 0 & 2 & 3 & 4 & 4 & 2 & 1 & 2 & 3 & 14 & 18 & 12 \\
\hline $\begin{array}{l}\text { Partidos regionales e } \\
\text { intereses específicos }\end{array}$ & 0 & 0 & 0 & 0 & 0 & 1 & 1 & 1 & 1 & 2 & 2 & 3 & 1 & 2 & 6 \\
\hline Total & 45 & 45 & 57 & 57 & 57 & 57 & 57 & 57 & 57 & 57 & 57 & 57 & 57 & 57 & 57 \\
\hline
\end{tabular}

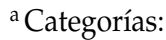

1) Partido de centro: se trata del Partido Liberación Nacional, la agrupación política de mayor trayectoria en el país. El PLN fundado en 1951 emergió como una agrupación de inclinación socialdemócrata. Sin embargo posteriormente modificó su carácter ideológico y actualmente constituye un partido centrista.

2) Partidos de derecha y centro/derecha: incluye a los partidos Demócrata, Republicano Nacional Independiente, Unión Nacional, Republicano, Independiente, Unión Cívico Revolucionaria, Unificación Nacional, Demócrata Cristiano, Nacional Independiente, Renovación Democrática, Coalición Unidad, Movimiento Nacional, Unidad Social Cristiana y Movimiento Libertario.

3) Partidos de izquierda y centro/izquierda: agrupa a los partidos Acción Demócrata Popular, Acción Socialista, Frente Popular Costarricense, Pueblo Unido, Alianza Popular, Fuerza Democrática, Acción Ciudadana y Frente Amplio.

4) Partidos regionales e intereses específicos: Unión Agrícola Cartaginés, Acción Democrática Alajuelense, Unión Generaleña, Agrario Nacional, Renovación Costarricense, Acción Laborista Agrícola, Integración Nacional, Accesibilidad sin Exclusión y Restauración Nacional.

Fuente: Elaboración propia con información del TSE.

\subsection{Número efectivo de partidos y volatilidad ${ }^{5}$}

En la literatura especializada sobre partidos políticos predomina la idea de que a mayor fragmentación del sistema político mayor dificultad para construir mayorías y que éstas generen acuerdos (Mainwaring, 1993). Para medir la fragmentación del sistema de partidos políticos se utiliza el indicador denominado Índice número efectivo de partidos (Laakso y Tagepera, 1979). Básicamente, entre más se acerque el valor del índice a 2 se está en presencia de un sistema de partidos catalogado como bipartidista y entre mayor el valor,

5 Para el cálculo del índice de número efectivo de partidos se utiliza la fórmula $\mathrm{N}=1 / \Sigma(\mathrm{S})^{2}$, donde (S) es el porcentaje de votos y/o escaños que obtiene cada partido político. Por su parte la volatilidad electoral se calcula mediante la fórmula $\mathrm{V}=\left(\sum 1 \% \mathrm{Vt} 1-\% \mathrm{Vt} 01\right) / 2$, en la que \% Vt1 es el porcentaje de votos obtenido por un partido político en la elección $\mathrm{t} 1$ (por ejemplo 2006) y \% Vt0 es el porcentaje de votos de ese mismo partido en la elección t0 (2002). 
mayor es la fragmentación del sistema. En el caso de Costa Rica, el valor del indicador de la elección legislativa ha sido mayor que en la elección presidencial desde 1978.

En el caso de las elecciones legislativa y municipal, en 2010 el sistema de partidos costarricense arrojó la mayor fragmentación del sistema político en 57 años. Luego del periodo bipartidista (1986-1998) las últimas tres elecciones reflejan un incremento de la fragmentación partidaria. Este aspecto se refleja en una composición pluripartidista y sin mayorías del Congreso y las municipalidades producto de una mayor cantidad de representantes políticos provenientes de distintas agrupaciones (Gráfico 1).

El otro aspecto analizado es la volatilidad electoral, que captura los cambios en las preferencias electorales entre partidos políticos (Pedersen, 1979). En 2010 este indicador disminuyó especialmente en las elecciones presidenciales, situación que debe ser interpretada como un efecto agregado de menor traslado de votos entre agrupaciones políticas, particularmente en el sentido PLN hacia otros partidos. Los cambios en las preferencias partidarias del 2010 reportados por el índice son ligeramente mayores que los registrados durante el bipartidismo, pero no llega a los niveles de los comicios de 1958, 1966 y 1978 (Gráfico 2).

Lo interesante del 2010 es que a diferencia de otras elecciones, un cambio en la volatilidad en la preferencia electoral no provoca una alteración en la fragmentación del sistema de partidos. Se esperaría que entre ambos indicadores se diera una relación directa, es decir, que a menor volatilidad menor la fragmentación, pues es menor la cantidad de electores que cambian su preferencia entre una elección y otra y por ende, menos partidos políticos conquistarían escaños. Sin embargo, este no es el caso.

Gráfico 1: Número efectivo de partidos en las distintas elecciones 1953-2010

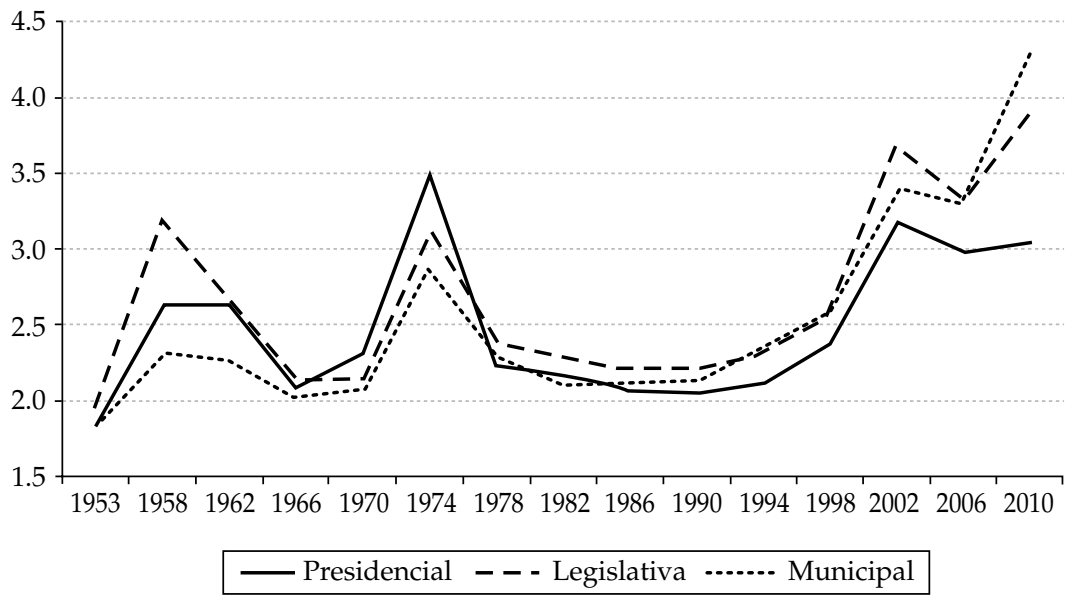

Fuente: Elaboración propia con datos del TSE. 
Gráfico 2: Volatilidad electoral según tipo de elección 1958-2010

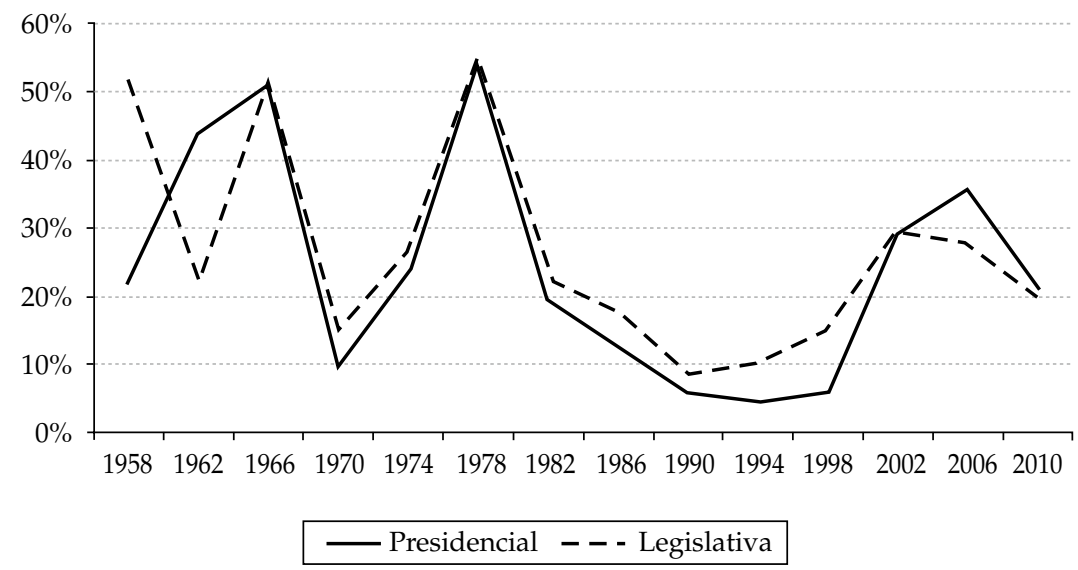

Nota: la volatilidad electoral se calcula mediante el índice de Pedersen que se obtiene sumando el cambio neto absoluto en el porcentaje de votos para cada partido de una elección a la otra y dividiéndolo por dos.

Fuente: Elaboración propia con datos del TSE.

\subsection{Amplio dominio del PLN en las alcaldías municipales}

A finales del 2010 se nombraron 81 alcaldes, 162 vicealcaldes, 944 síndicos municipales propietarios y suplentes, 3.712 concejales de distrito propietarios y suplentes, 64 concejales municipales de distrito propietarios y suplentes, 8 intendentes y 8 viceintendentes (Cuadro 4 ).

Las elecciones municipales del 2010 reafirmaron la tendencia al desalineamiento político de la ciudadanía costarricense y la fragilidad del sistema de partidos emergente. Como consecuencia, en los ayuntamientos predominan, en la última década, instancias pluripartidistas y sin mayorías políticas. Por otra parte, solo una cuarta parte de los empadronados emitió su voto en los comicios locales de 2010, una cifra semejante a la de 2002 y 2006. Esto apunta a la existencia de un "techo" en la participación electoral a nivel local que puede ser considerado bajo si se le compara con las elecciones nacionales y desde una perspectiva internacional comparada.

El Código Electoral aprobado en 2009 amplió el financiamiento público a las elecciones municipales desprovistas de tales recursos. De acuerdo con el artículo 91 del Código Electoral de 2008, "el Estado contribuirá con un 0,03\% del PIB para cubrir los gastos en que incurran los partidos políticos con derecho a ellos por su participación en los procesos electorales de carácter municipal, de conformidad con las reglas establecidas en la Constitución Política y en este Código". Según la legislación electoral en su artículo 99, los partidos que participan en elecciones municipales podrán tener derecho al financiamiento público si alcanzan al menos un $4 \%$ de los votos válidamente emitidos en el cantón respectivo para la elección de alcalde o regidores, o bien, si eligen al menos un regidor o regidora. 
Cuadro 4: Cifras relevantes de las elecciones municipales. 2006 y 2010

\begin{tabular}{lrrl}
\hline Componente & \multicolumn{1}{c}{2006} & \multicolumn{1}{c}{2010} & Comportamiento \\
\hline Padrón electoral & 2.603 .770 & 2.866 .217 & Aumentó \\
Juntas receptoras de votos & 4.852 & 5.250 & Aumentó \\
Partidos políticos participantes & 45 & 46 & Aumentó \\
Cargos por elegir & 4.941 & 4.971 & Aumentó \\
Candidaturas inscritas & 15.919 & 15.862 & Disminuyó \\
Alcaldes & 81 & 81 & Igual \\
Vicealcaldes & 162 & 162 & Igual \\
Síndicos (propietarios y suplentes) & 938 & 944 & Aumentó \\
Concejales de distrito (propietarios & & & \\
y suplentes) & 3.688 & 3.712 & Aumentó \\
Intendentes & 8 & 8 & Igual \\
Concejales municipales de distrito & & & \\
(propietarios y suplentes) & 64 & 64 & Igual \\
Viceintendentes & & 8 & Cargo no existía antes de 2010 \\
\hline
\end{tabular}

Fuente: Elaboración propia con datos del TSE.

\subsection{Predominio del PLN en las alcaldías y concejos municipales divididos}

Los dos principales resultados de las elecciones municipales fueron, por una parte, que el PLN ratificó su hegemonía en las alcaldías que mantiene desde el 2006, y por otra, que no hay un claro control partidario de los Concejos Municipales debido a la alta volatilidad y la pluralidad de agrupaciones que los conforman. El oficialismo ganó 59 de las 81 en disputa, lo que representa el 73\% de los gobiernos locales. Muy por debajo le siguió el PUSC con sólo nueve alcaldías y el PAC con seis. En el caso de estos dos últimos hubo cambios con respecto al 2006. El PUSC perdió control sobre las alcaldías, pues pasó de elegir 11 alcaldes en 2006 a 9 en las más recientes de 2010, mientras que el PAC más bien recuperó terreno al pasar de 4 alcaldes en 2006, a 6 en diciembre pasado (Cuadro 5).

En el caso de los partidos Movimiento Libertario y Acción Ciudadana (2 y 6 alcaldías, respectivamente), su respaldo en los comicios municipales disminuyó de manera considerable en comparación con el registrado en las elecciones nacionales de febrero, lo cual revela la incapacidad para retener a sus partidarios y armar una organización territorial para hacerle frente a una contienda electoral de carácter local.

Desde una perspectiva comparada, esta distribución del poder en las municipalidades se asemeja a la del 2002, elección en la que los resultados de los comicios nacionales pusieron fin a 16 años de era bipartidista. Los alcaldes en gobiernos divididos estarán obligados a realizar mayores esfuerzos de negociación y apertura para alcanzar acuerdos. Si esto no prospera, existe la amenaza de serios problemas en la gestión de los asuntos locales, con alcaldes incapaces de ejecutar sus programas políticos por la obstrucción de la oposición en el Concejo. Ahora bien, la existencia de gobiernos divididos no puede ser considerada 
Cuadro 5: Resultados de las elecciones de alcaldes municipales, según partido político. 2006 y 2010

\begin{tabular}{lrrrrrrrr}
\hline \multirow{2}{*}{ Partido político } & \multicolumn{3}{c}{2006} & & \multicolumn{3}{c}{2010} \\
\cline { 2 - 3 } \cline { 8 - 9 } & Votos & Porcentaje & Alcaldes & & Votos & Porcentaje & Alcaldes \\
\hline Liberación Nacional & 277.589 & 45,9 & 59 & & 342.563 & 42,9 & 59 \\
Unidad Social Cristiana & 107.007 & 17,7 & 11 & & 113.394 & 14,2 & 9 \\
Acción Ciudadana & 88.630 & 14,6 & 4 & & 98.719 & 12,4 & 6 \\
Movimiento Libertario & 34.073 & 5,6 & 1 & & 75.441 & 9,4 & 2 \\
Otros partidos & 97.896 & 16,2 & 6 & & 169.054 & 21,2 & 5 \\
Total & 605.195 & 100 & 81 & & 799.171 & 100 & 81 \\
\hline
\end{tabular}

a La categoría "Otros partidos" incluye 39 agrupaciones en 2006 y 42 en el 2006.

Fuente: elaboración propia con datos del TSE.

como negativa para la democracia. La división partidaria y la necesidad de construir acuerdos también pueden generar dinámicas políticas más abiertas y transparentes, con un Concejo de oposición pidiendo cuentas y vigilando más de cerca la labor del alcalde que en los casos en los que un mismo partido controla ambos órganos.

\section{PODER EJECUTIVO}

Los primeros dos años de gestión del Ejecutivo (2010-2011) fueron atípicos. Cuando apenas iniciaba la nueva Administración se dieron inusitadas divisiones dentro del partido oficialista (PLN) que debilitaron al Poder Ejecutivo, tanto en su relación con el Legislativo como en su capacidad para conducir el aparato institucional. Tres eventos afectaron la gestión de los asuntos públicos: el primero fue iniciar el año legislativo con un proyecto de ley para aumentar el salario de los diputados, proyecto que tuvo el apoyo de la bancada de legisladores oficialistas y el beneplácito del Ejecutivo, y que finalmente fue archivado ante la crítica de los partidos de oposición y la opinión pública.

El segundo aspecto fueron las aspiraciones electorales tempraneras dentro del PLN de cara a las presidenciales de 2014. Ciertamente la postulación de nombres dentro de los partidos no encierra en sí mismo un problema. Lo que resulta inusual es que, tan temprano en una nueva administración, estas diferencias emerjan con tanta fuerza y que además dividan el bloque de legisladores del partido de gobierno. Tradicionalmente el primer año es más calmo y hay más espacio para "acomodarse" e implementar las principales líneas de trabajo de la nueva administración, pero ese no fue el caso durante el 2010 e inicios del 2011.

El tercer aspecto está relacionado con la alta rotación en puestos de mando, sobre todo ministerios y viceministerios. Solo en los primeros 15 meses de gestión ocho ministros dejaron su puesto o fueron sustituidos, y hasta abril de 2012 ya son doce los cambios. Entre ellos destaca la salida de la ministra de Salud por diferencias con la Presidencia 
en la conducción de la crisis financiera y administrativa del sistema de seguridad social (CCSS), el cambio en la jefatura del Ministerio de Relaciones Exteriores por el manejo del conflicto con Nicaragua y las contrataciones en el Servicio Exterior, y más recientemente la renuncia del ministro de Hacienda por denuncias de irregularidades en sus declaraciones de impuestos (Cuadro 6).

Cuadro 6. Cambios en el Gobierno durante el 2011 y 2012 según institución/sector

\begin{tabular}{|c|c|c|c|}
\hline Institución/Sector & Saliente & Cambio & $\begin{array}{l}\text { Fecha del } \\
\text { cambio }\end{array}$ \\
\hline \multicolumn{4}{|l|}{ Ministerios } \\
\hline Deportes & Guiselle Goyenaga & William Todd & $08 / 02 / 2011$ \\
\hline Ciencia y Tecnología & Clotilde Fonseca & Alejandro Cruz & $15 / 02 / 2011$ \\
\hline Turismo & Carlos Ricardo Benavides & Allan Flores & $04 / 04 / 2011$ \\
\hline Presidencia & Marco Vargas & Carlos Ricardo Benavides & 04/04/2011 \\
\hline Seguridad & José María Tijerino & Mario Zamora & $30 / 04 / 2011$ \\
\hline Ambiente & Teófilo de la Torre & Reneé Castro & $25 / 07 / 2011$ \\
\hline Relaciones Exteriores & René Castro & Enrique Castillo & $31 / 07 / 2011$ \\
\hline Salud & María Luisa Ávila & Daisy Corrales Díaz & $05 / 08 / 2011$ \\
\hline Deportes & William Todd & -Vacante- & $10 / 01 / 2012$ \\
\hline Planificación & Laura Alfaro & Roberto Gallardo & $15 / 03 / 2012$ \\
\hline Comunicación & Roberto Gallardo & Francisco Chacón & $15 / 03 / 2012$ \\
\hline Hacienda & Fernando Herrero & Edgar Ayales & $17 / 04 / 2012$ \\
\hline \multicolumn{4}{|l|}{ Viceministerios } \\
\hline Seguridad & Flora María Calvo & Xinia Montano & $20 / 01 / 2011$ \\
\hline Planificación & Gaudy Solórzano & Melania Núñez & 08/02/2011 \\
\hline Relaciones Exteriores & Marta Núñez Madriz & Fernando Salazar Alvarado & $29 / 04 / 2011$ \\
\hline Seguridad & Mario Zamora & Celso Gamboa & $30 / 04 / 2011$ \\
\hline Trabajo & Álvaro Sojo & José Manuel Cordero & $31 / 05 / 2011$ \\
\hline Transportes & Maristella Vaccari & Rodrigo Rivera & $31 / 05 / 2011$ \\
\hline Hacienda & Jenny Phillips & Rowland Espinoza & $31 / 05 / 2011$ \\
\hline Ciencia y Tecnología & Rowland Espinoza & Keilor Rojas & $21 / 06 / 2011$ \\
\hline Ambiente & Andrei Bourrouet & María Guzmán Ortiz & $01 / 02 / 2012$ \\
\hline \multicolumn{4}{|l|}{ Instituciones autónomas } \\
\hline AyA & Oscar Núñez & Yessenia Calderón & $22 / 07 / 2011$ \\
\hline ICE & Eduardo Doryan & Teófilo de la Torre & 09/08/2011 \\
\hline \multicolumn{4}{|l|}{ Diputados oficialistas } \\
\hline PLN & Guillermo Zúñiga & Antonio Calderón & $01 / 05 / 2011$ \\
\hline PLN & Francisco Chacón & Carolina Delgado & $15 / 03 / 2012$ \\
\hline PLN & Viviana Martin & Jorge Arturo Rojas & $23 / 03 / 2012$ \\
\hline
\end{tabular}

Fuente: Elaboración propia con base en prensa escrita y digital 
La gestión de la Presidenta también se ha deteriorado en los últimos dos años con una reducción de los apoyos sobre la labor de la Presidenta y del gobierno en general. Bajó el índice de confianza en el Gobierno, la percepción de la labor del Gabinete, y de manera más estrepitosa, las percepciones sobre el liderazgo de la Presidenta (Cuadro 7). En esta última variable pasó de un $74 \%$ de los encuestados que reconocían el liderazgo de la mandataria Chinchilla en Julio de 2010, a tan solo 13\% en Enero de 2012.

\section{PODER LEGISLATIVO}

Como se indicó anteriormente, el primer año de la administración Chinchilla Miranda estuvo caracterizado por importantes desgastes del oficialismo que fue aprovechado por la oposición en el Congreso. Por primera vez en la historia reciente del país, se conformó un Directorio Legislativo de oposición -algo que no sucedía desde 1969-, fiel reflejo del debilitamiento político que sufrió el PLN en la primera legislatura.

Las estadísticas muestran un Congreso más efectivo en cuanto a la cantidad de leyes aprobadas comparado con las últimas dos administraciones (Cuadro 8). No obstante, cuando se trata de piezas de legislación clave para el país, el sistema se muestra incapaz de articular intereses y sacar adelante los proyectos requeridos.

Cuadro 7. Evolución de las percepciones sobre el Gobierno, en porcentajes

\begin{tabular}{|c|c|c|c|c|c|c|}
\hline Variable & $\begin{array}{l}\text { Julio } \\
2010\end{array}$ & $\begin{array}{l}\text { Octubre } \\
2010\end{array}$ & $\begin{array}{c}\text { Marzo } \\
2011\end{array}$ & $\begin{array}{c}\text { Junio } \\
2011\end{array}$ & $\begin{array}{c}\text { Octubre } \\
2011\end{array}$ & $\begin{array}{l}\text { Enero } \\
2012\end{array}$ \\
\hline Índice de confianza en el Gobierno ${ }^{a}$ & 2,81 & 2,25 & 2,11 & 1,55 & 1,47 & 1,29 \\
\hline Labor del gabinete ${ }^{b}$ & 27 & 22 & 22 & 13 & 14 & 11 \\
\hline Eficiencia gasto público $^{c}$ & 45 & 39 & 28 & - & - & 20 \\
\hline $\begin{array}{l}\text { Capacidad del Gobierno para resolver los } \\
\text { problemas del país }^{\mathrm{d}}\end{array}$ & 58 & 46 & 39 & 30 & 32 & - \\
\hline Labor de presidenta $^{\mathrm{b}}$ & 38 & 41 & 37 & 26 & 26 & 23 \\
\hline Liderazgo de presidenta $^{\mathrm{e}}$ & 74 & - & 58 & 14 & - & 13 \\
\hline Labor de la Asamblea Legislativab & 20 & 21 & 15 & 7 & 8 & - \\
\hline Simpatiza con ningún partido ${ }^{f}$ & 39 & 42 & 51 & 42 & - & - \\
\hline Principal problema: Inseguridad/delincuencia & 46 & 33 & 38 & 49 & - & 28 \\
\hline
\end{tabular}

a Se calcula en una escala de 1 a 5, donde 1 es la más baja confianza y 5 la más alta.

b Se reportan datos de buena y muy buena.

' Se reportan datos de: eficiencia y cierta eficiencia.

d Se reportan datos de: sabe como resolver el problema pero necesita más tiempo.

e Se reportan respuestas afirmativas.

${ }^{\mathrm{f}} \mathrm{Se}$ reportan datos para la categoría de ninguno.

Fuente: Elaboración propia con datos de Unimer. 
Cuadro 8. Leyes aprobadas en la primera legislatura, por administración, según tipo de leyes. 2002-2011

\begin{tabular}{|c|c|c|c|c|c|c|}
\hline \multirow{2}{*}{ Tipo de leyes } & \multicolumn{2}{|c|}{$\begin{array}{c}\text { Pacheco } \\
2002 / 2003\end{array}$} & \multicolumn{2}{|c|}{$\begin{array}{c}\text { Arias } \\
2006 / 2007\end{array}$} & \multicolumn{2}{|c|}{$\begin{array}{l}\text { Chinchilla } \\
\text { 2010/2011 }\end{array}$} \\
\hline & Leyes & Porcentaje & Leyes & Porcentaje & Leyes & Porcentaje \\
\hline Autorizaciones locales & 30 & 40,5 & 46 & 61,3 & 31 & 27,4 \\
\hline Convenios y tratados internacionales & 14 & 18,9 & 10 & 13,3 & 39 & 34,5 \\
\hline Declaraciones y benemeritazgos & 1 & 1,4 & 0 & 0,0 & 8 & 7,1 \\
\hline Legislación sustantiva ordinaria & 27 & 36,5 & 17 & 22,7 & 32 & 28,3 \\
\hline Presupuestos & 2 & 2,7 & 2 & 2,7 & 3 & 2,7 \\
\hline Totales & 74 & 100,0 & 75 & 100,0 & 113 & 100,0 \\
\hline
\end{tabular}

Fuente: Feoli, 2011.

El proceso de aprobación de las leyes sustantivas es largo, lo cual también dificulta la ejecución de política pública por parte del Ejecutivo cuando éste depende de decisiones parlamentarias. La legislación sustantiva, que está directamente relacionada con aspectos del desarrollo humano en el país, dura en promedio entre dos años y medio y tres años en ser aprobada (Cuadro 9). Esta situación plantea serias dificultades de gestión para un gobierno, pues el periodo presidencial en Costa Rica dura cuatro años. Eso significa que los proyectos de ley prioritarios para el Ejecutivo podrían ser aprobados sólo a partir de la mitad del periodo, y la ejecución real de esos proyectos puede trascender el mandato presidencial. Ello también explica por qué, durante los primeros dos años de cada nuevo gobierno, la gran mayoría de iniciativas que se aprueban en el Congreso son de administraciones anteriores.

Finalmente, al revisar los proyectos de ley más importantes en el Congreso y discutidos por la opinión pública desde mayo de $2010^{6}$, se pueden identificar al menos cinco: el aumento salarial de los diputados; la reforma a la Ley de Tránsito, la reforma fiscal, el proyecto de Ley General de Electricidad y la reforma al artículo 78 de la Constitución para asignar el 8\% del PIB a la educación. Estas iniciativas no solo han sido relevantes para los formadores de opinión del país, sino que además fueron parte de las prioridades establecidas por el gobierno de la Presidenta Chinchilla a lo largo de este período.

De ellos, solo dos han tenido alguna definición de parte del Congreso. Se trató del expediente que pretendía un alza del 72\% en el salario de los diputados, que como ya se indicó fue archivado. El otro proyecto, y único de esta lista que ha logrado ser aprobado, es la reforma constitucional para asignar el 8\% del PIB a la educación, antes explicado también.

Los otros tres proyectos aún siguen en trámite. De ellos, la reforma fiscal es el mejor ejemplo del largo y complejo proceso legislativo. A pesar del reconocimiento generalizado 
Cuadro 9. Duración promedio en meses del proceso de aprobación de leyes, por legislatura ${ }^{a}$

\begin{tabular}{lcc}
\hline Leyes aprobadas & $2010-2011$ & $2011-2012^{\mathrm{b}}$ \\
\hline Autorizaciones locales & 26,7 & 26,0 \\
Convenios y tratados internacionales & 30,5 & 23,1 \\
Declaraciones y benemeritazgos & 20,6 & 32,4 \\
Derogación de leyes & - & 22,2 \\
Legislación sustantiva ordinaria & 32,1 & 36,4 \\
Presupuestos & 2,1 & 3,0 \\
Total & 28,4 & 29,9 \\
\hline
\end{tabular}

aLa legislatura inicia el 01 de mayo y termina el 30 de abril del año siguiente.

bDatos hasta febrero de 2012.

Fuente: Elaboración propia con información de la Asamblea Legislativa.

de la necesidad de una reforma fiscal, no se ha logrado la convergencia de intereses entre los partidos representados en la Asamblea Legislativa. El Ejecutivo, que diseñó y puso a discusión del Congreso el proyecto de ley desde inicios del 2011, aparece como uno de los principales responsables por no fomentar un diálogo abierto entre partidos en esta materia (Programa Estado de la Nación: 2011). Y el panorama se vuelve más sombrío luego de que el proyecto fuera declarado inconstitucional por la Sala Constitucional.

\section{EVALUACIÓN GENERAL SOBRE EL FUNCIONAMIENTO Y CALIDAD DE LA DEMOCRACIA}

El periodo 2010-2011 muestra dos aspectos centrales sobre el funcionamiento y la calidad de la democracia: por un lado una democracia electoral sólida, con buenos niveles de control y transparencia que garantizan estabilidad política y legitimidad al proceso de competencia electoral. Por otro lado, se evidencian importantes deficiencias del sistema político para gestionar adecuadamente los temas más importantes del país en materia de desarrollo humano y traducirla en política pública bajo la coyuntura actual.

Estos problemas de gestión confluyen con un contexto de cambio y diversificación de los partidos representados en el Congreso y a nivel local. Y aunque esta aparición de nuevas agrupaciones representadas es un buen indicador de pluralidad política, también es cierto que pone sobre el sistema político en general, y sobre los líderes políticos en particular, mayores responsabilidades para la ejecución de la política pública.

En este escenario, se requiere de más y mejor calidad en la deliberación política, con élites gobernantes capaces de negociar antes que imponer. Sobre todo porque la distribución actual de fuerzas no le permite a un solo grupo definir el rumbo del país. Ningún partido tiene, en el Congreso por ejemplo, los votos suficientes para tomar 
decisiones sin necesidad de negociar con la oposición. Pero el país ciertamente necesita de consensos renovados entre las principales fuerzas políticas. Así fue como se lograron dirimir las tensiones después de la guerra civil de 1948 que decantó en la construcción de un Estado de bienestar y sin ejército. Hoy en día el país requiere un diálogo similar que lleve a solventar los problemas de desigualdad e inseguridad crecientes y permita encontrar puntos intermedios en temas sensibles como la reforma fiscal, infraestructura, y la seguridad social.

Con ese marco de fondo, de cara al 2012 y 2013 será vital el desenlace de los grandes temas de discusión del país, y más importante aún, la forma como la institucionalidad democrática responde ante ellos. En particular la discusión sobre la situación fiscal y el paquete de reformas tributarias que busca dotar de más recursos al Estado. Esta es una ventana de oportunidades para que la élite gobernante concrete acuerdos de largo plazo y manden una señal positiva al sistema político y al país en general.

\section{REFERENCIAS}

Alfaro-Redondo, Ronald. 2006. "Elecciones nacionales 2006 en Costa Rica y la recomposición del sistema de partidos políticos". Revista de Ciencia Política (Santiago) 26: 125-37.

Birnir, Jóhanna and Donna Lee Van Cott. 2007. "Disunity in Diversity: Party System Fragmentation and the Dynamic Effect of Ethnic Heterogeneity on Latin American Legislatures". Latin American Research Review 42, no. 1: 99-125.

Booth, John A., Christine J. Wade, and Thomas W. Walker. Understanding Central America: Global Forces, Rebellion, and Change. 5th ed. Boulder, CO: Westview Press.

Feoli, Ludovico. 2011. "Desempeño Legislativo en la primera legislatura de la administración Chinchilla". Ponencia preparada para el Decimosétimo Informe Estado de la Nación. San José, Programa Estado de la Nación.

2009. "Costa Rica Después Del TLC: ¿La Calma que Sigue a la Tempestad?". Revista de Ciencia Política (Santiago) 29: 355-79.

Gómez, Steffan y Villarreal, Evelyn. 2010. “Costa Rica 2009: Enfrentando la crisis internacional en plena campaña electoral". Revista de Ciencia Política, Pontificia Universidad de Chile, pp. 275-296. Santiago de Chile.

Hernández R., Oscar. 1991. “Análisis del Abstencionismo en las Elecciones Presidenciales de Costa Rica en el período 1953-1986". Anuario de Estudios Centroamericanos 16-17, no. 2-1 (1990-1991): 117-37.

Granados, Carlos and Ohlsson, Anita. 1983. "Organización del Territorio y Resultados Electorales en Costa Rica, 1953-1982". Estudios Sociales Centroamericanos 12, no. 36: 183-210.

Jones, Mark P., and Scott Mainwaring. 2003. "The Nationalization of Parties and Party Systems." Party Politics 9, no. 2 (March 1): 139-66.

Laakso, Markku and Rein Taagepera. 1979. "Effective Number of Parties: A Measure with Application to West Europe". Comparative Political Studies 12, no. 1 (April 1): 3-27.

Lapop, et al. 2005. "La Cultura Política de la Democracia en Costa Rica: Un Estudio Del Proyecto De Opinión Pública en América Latina (Opal)". Americas Barometer. Latin American Public Opinion Project (Lapop); Universidad de Costa Rica, Centro Centroamericano de Población (CCCP-UCR); United States Agency for International Development (USAID). Costa Rica.

Lehoucq, Fabrice Edouard. 1995. "The Costa Rican General Elections of 6 February 1994". Electoral Studies 14, no. 1: 69-72.

1997. Lucha Electoral y Sistema Político en Costa Rica, 1948-1998. Colección Pasado y Presente. 1a. ed. San José, Costa Rica: Editorial Porvenir. 
Lehoucq, Fabrice, Iván Molina Jiménez, and Ebrary Inc. 2002. Stuffing the Ballot Box Fraud, Electoral Reform, and Democratization in Costa Rica. New York: Cambridge University Press: http:/ / www. columbia.edu/cgi-bin/cul/resolve?clio5389945.

Mainwaring, Scott. 1993. "Presidentialism, Multipartism, and Democracy: The Difficult Combination". Comparative Political Studies 26, no. 2 (July 1): 198-228.

Mitchell A., Seligson. 2002. "Trouble in Paradise?: The Erosion of System Support in Costa Rica, 19781999". Latin American Research Review 37, no. 1: 160-85.

Mitchell A., Seligson y Gómez Barrantes, Miguel. 1987. “Elecciones Ordinarias en Tiempos Extraordinarios: La Economía Política del Voto en Costa Rica". Anuario de Estudios Centroamericanos 13, no. 1: 5-24.

Molina Jiménez, Iván. 2001. "Democracia y Elecciones en Costa Rica: Dos Contribuciones Polémicas". Cuadernos de Ciencias Sociales. 1a. ed. San José, Costa Rica: Facultad Latinoamericana de Ciencias Sociales, Sede Académica, Costa Rica.

Pedersen, Mogens N. 1979. "The Dynamics of European Party Systems: Changing Patterns of Electoral Volatility". European Journal of Political Research 7, no. 1: 1-26.

Programa Estado de la Nación. 2001. Auditoría Ciudadana Sobre la Calidad de la Democracia. 1a. ed. Pavas, Costa Rica: Proyecto Estado de la Nación, 2001.

2010. Decimosexto Informe Estado de la Nación. San José, Programa Estado de la Nación. 2011. Decimoséptimo Informe Estado de la Nación. San José, Programa Estado de la Nación.

Raventos Vorst, Ciska. 2005. Abstencionistas en Costa Rica: Quiénes Son y Por Qué no Votan? 1a. ed. San José, Costa Rica: Editorial de la Universidad de Costa Rica: Tribunal Supremo de Elecciones: IIDH/ CAPEL: Instituto de Investigaciones Sociales.

Rojas Bolaños, Manuel y Carlos Sojo. 1995. El Malestar con la Política: Partidos y Élites en Costa Rica. 1a. ed. San José, Costa Rica: FLACSO, Programa Costa Rica.

Rojas Bolaños, Manuel. 1989. La Democracia Inconclusa. Colección Universitaria. Universidad de Costa Rica, Instituto de Investigaciones Sociales, y Departamento Ecuménico de Investigaciones (Costa Rica). San José.

Rovira Mas, Jorge. 2000. Estado y Política Económica en Costa Rica, 1948-1970. 2. ed. San José: Editorial de la Universidad de Costa Rica.

Rovira Mas, Jorge y Mario Sánchez Machado. 1986. La Crisis de Costa Rica. Proyecto Crisis y Alternativas en Centroamérica. Documentos de Trabajo. Instituto Centroamericano de Documentación e Investigación Social (ICADIS). San José.

Rovira Mas, Jorge. 2001. La Democracia de Costa Rica ante el Siglo XXI. Universidad de Costa Rica. Instituto de Investigaciones Sociales.1a. ed. San José, Costa Rica: Editorial de la Universidad de Costa Rica.

Salazar Mora, Orlando y Jorge Mario Salazar Mora. 1991. Los Partidos Políticos en Costa Rica. 1a. ed. San José, Costa Rica: Editorial Universidad Estatal a Distancia.

Salom E, Roberto. 1987. La Crisis de la Izquierda en Costa Rica. Colección Debate. 1a. ed. San José, Costa Rica: Editorial Porvenir.

Sánchez, Fernando. 2002. “Cambios Políticos En Centroamérica: El Desalineamiento Electoral En Costa Rica". Bicentenario 1, no. 2: 117-146.

2004. Dealignment in Costa Rica: a Case Study of Electoral Change. Doctoral dissertation. University of Oxford. England.

Sánchez Machado, Mario. 1985. Las Bases Sociales del Voto en Costa Rica, 1974-1978. 1a. ed. San José, Costa Rica: Uruk Editores.

Seligson, Mitchell A. 1980. Peasants of Costa Rica and the Development of Agrarian Capitalism. Madison: University of Wisconsin Press.

Urcuyo Fournier, Constantino. 2003. Reforma Política y Gobernabilidad. San José, Costa Rica: Editorial Juricentro.

Vargas Cullell, Jorge. 2007. "Costa Rica: Fin de una era Política". Revista de Ciencia Política (Santiago) 27: 113-28.

2008. "Costa Rica: Una Decisión Estratégica en Tiempos Inciertos". Revista de Ciencia Política (Santiago) 28: 147-69. 
Villalobos, Carlos. 2011. "Inseguridad se dispara como principal problema en el país". La Nación, 4 julio. Sección El País. San José.

2011. "Diputados cierran año con deuda en tres prioridades del Gobierno". La Nación, 29 de Abril, Sección El País. San José.

Wills-Otero, L. 2009. Electoral Systems in Latin America: Explaining the Adoption of Proportional Representation Systems During the Twentieth Century. 33, 2009.

Ronald Alfaro-Redondo. Costarricense. Politólogo, investigador y docente universitario. Máster en Métodos Cuantitativos en las Ciencias Sociales de la Universidad de Columbia en Nueva York, USA. Estudiante de doctorado en Ciencia Política de la Universidad de Pittsburgh, Pennsylvania, USA. Autor principal del estudio del Barómetro de las Américas para Costa Rica. Investigador del Capítulo Fortalecimiento de la Democracia del Programa Estado de la Nación. Profesor de la Escuela de Ciencias Políticas de la UCR en las áreas de metodología de la investigación y realidad nacional. Sus áreas de trabajo son la política comparada en América Latina: elecciones y sistemas electorales, partidos políticos; participación política y cultura política.

E-mail: ralfaro@estadonacion.or.cr.

Steffan Gómez-Campos. Costarricense. Politólogo, investigador y docente universitario. Máster en Estudios del Desarrollo por la Jüstus Liebig Universität de Giessen en Alemania, y Licenciado con honores en Ciencias Políticas por la Universidad de Costa Rica (UCR). Coordinador de investigación del Capítulo Fortalecimiento de la Democracia del Programa Estado de la Nación. Profesor de la Escuela de Ciencias Políticas de la UCR en las áreas de comunicación política, metodología de la investigación y realidad nacional. Sus áreas de trabajo son la política comparada en América Latina: democracia y desarrollo; gobernabilidad; elecciones; reformas políticas y electorales; y cultura política. E-mail: sgomez@estadonacion.or.cr. 